Saudi Journal of Oral and Dental Research

Abbreviated Key Title: Saudi J Oral Dent Res

ISSN 2518-1300 (Print) |ISSN 2518-1297 (Online)

Scholars Middle East Publishers, Dubai, United Arab Emirates

Journal homepage: https://saudijournals.com

Review Article

\title{
Oro-Antral Communication and Fistula: A Review of the Literature
}

\author{
${\text { Bader Fatani }{ }^{1 *} \text {, Ahmed Fatani }{ }^{2} \text {, Ahmed Alomar }}^{3}$
}

${ }^{1}$ College of dentistry King Saud University Saudi Arabia

${ }^{2}$ General Dentist ministry of health Saudi Arabia

${ }^{3}$ Associate consultant Oral Maxillofacial Surgery King Saud University Saudi Arabia

DOI: $\underline{10.36348 / \text { sjodr.2020.v05i12.002 }}$ | Received: 20.11.2020 | Accepted: 03.12.2020 | Published: 05.12 .2020

*Corresponding author: Bader Fatani

\section{Abstract}

Oro-antral communication is an abnormal connection between the maxillary sinus and the oral cavity. This communication can occur due to pathological or non-pathological reasons. However, it is more common after the extraction of the maxillary posterior teeth which have their root tips close or attached to the maxillary sinus. Spontaneous healing is usually seen in OAC with less than $2 \mathrm{~mm}$ in diameter. However, in case the OAC is more than $3 \mathrm{~mm}$ a surgical treatment is usually considered to avoid further complications such as sinusitis. In this review, we will illustrate the appropriate diagnosis of the OAC and discuss all the surgical options for the closure of the OAC including the local flaps with the advantages and disadvantages of each technique.

Keywords: Oroantral fistula, buccal flap, buccal fat pad, palatal rotational flap.

Copyright () 2020 The Author(s): This is an open-access article distributed under the terms of the Creative Commons Attribution 4.0 International License (CC BY-NC 4.0) which permits unrestricted use, distribution, and reproduction in any medium for non-commercial use provided the original author and source are credited.

\section{Introduction to the Oro-Antral Communication}

Oroantral communication (OAC) is an abnormal rare connection which links between the oral cavity and the maxillary sinus $[1,10]$ it is generally caused by the perforation of roots tip into the maxillary sinus as a complication after extraction of maxillary posterior teeth [1]. The premolars and molars which have their roots tip approximated or attached to the maxillary sinus and parted by a thin bony lamella is a common site for this complication [6, 14]. OAC can also be associated with a variety of surgical approaches or undesirable events such as orthognathic surgery, tuberosity fracture, Caldwell-Luc procedure, sequelae of radiation therapy, osteomyelitis, trauma, cyst or carcinoma, tumor, implant, infection, sinusitis, and iatrogenic complications [1, 3, 9, 14, 15]. Oroantral fistula (OAF) which is the epithelialized form of the OAC is usually considered pathological since it is related to a specific disease or a bacteria which can cause infection of the antrum [12]. However, it can also be non-pathological since it can result from trauma during the extraction of the maxillary posterior teeth or after the removal of a dental implant $[1,2,12]$. OAF is considered a complex defect as it involves the hard and soft tissue layers [4]. Depending on the location of the OAF it can be classified as palatal-sinusal, alveolosinusal, and vestibulo-sinusal [11]. The Presence of tumors, foreign bodies, dental cysts, osteomyelitis or osteitis, dental apical abscess, or maxillary sinusitis in the communication area will result in restrain of the spontaneous healing process and the development of a chronic fistula [1]. The closure of the communication is essential to prevent food and saliva contamination which will lead to infection and impaired healing [11]. This study aims to give a general review of the $\mathrm{OAC} / \mathrm{OAF}$ and illustrate the appropriate decision in the closure of the OAF depending on the diagnosis of the fistula, postoperative complications, and the success rate of each treatment.

\section{Pre-operative diagnosis of the OAC}

An appropriate medical history is essential in determining the risk of developing a complication during or after the closure of the OAC [12]. Many factors contribute in making the best decision for the closure of the $\mathrm{OAC}$, this includes the presence of infection, availability of tissue for repairing the defect, position and dimension of the fistula, and the period in which the fistula was diagnosed. ${ }^{1}$ Patients with renal dysfunction, cardiac disease, and hematological disorder have a high risk of developing an infection, bleeding and delayed tissue healing [12]. The surgeon have to determine if the procedure should be immediate or delayed based on the health and thickness of the tissue around the defect and taking into account the patient health status[14]. The symptoms associated with OAF usually include a postnasal exudate, pain in the upper teeth, altered nasal resonance, whistling sound while speaking, halitosis, and a poor sense of taste and smell $[3,15]$. The patient may also complain of pain in 
the malar region [15]. Early diagnosis of the OAC is required for a better outcome [6]. Diagnosing of the OAC includes intraoral examination, cheek-blowing test, Valsalva test, and exploration of the perforation with probing [12]. In an intraoral examination, a large $\mathrm{OAC}$ can be easily seen during the investigation. However, at a later stage an antral polyp might be seen in the defect [12]. During the Valsalva test, the patient will try to exhale through the blocked nasal airway [12]. In the cheek blowing test, the patient should be instructed to blow air to his cheeks while the mouth is closed. The main problem of this examination that it can be associated with a possibility of antral complication by the transmission of microorganisms from the oral cavity to the maxillary sinus [12].

Clinical findings of the OAC may include air and fluid passing into mouth or nose, and unpleasant salty discharge [14]. A blood bubbles might be seen in the defect or the patient may feel a leakage of air when he blows while nostrils are closed [14]. Resonance of the sound and speech may also be experienced by the patient if the defect is large [14]. However, some patients may not present with these findings if the communication was too small or covered by a large polyp [14]. Radiographs such as panoramic radiograph or CT is used to examine the site and to confirm the clinical findings [14]. The panoramic radiograph is used to give a precise estimation of the dimension of the bony defect of the fistula and illustrate the location and presence of dental roots or any foreign body in the antrum [15]. The computed tomography is also done to exclude the presence of maxillary sinusitis [15]. Radiographs might represent sinus opacity, floor discontinuity, periodontal disease, and focal alveolar atrophy [11].

The fistula should be closed within 48 hours of onset to avoid further complications in the future [3]. Proper closure of the OAC is strongly associated with the condition of the maxillary sinus [14]. If the OAC is less than $2 \mathrm{~mm}$ and associated with normal blood coagulation level, absence of infection and without epithelization of the OAC, the communication usually ends in healing and resolving by secondary healing and blood clotting $[2,4]$. However, In case the OAC is more than $3 \mathrm{~mm}$, a surgical approach such as soft tissue and bone grafts, allograft, xenograft and other techniques like re-implantation of third molars should be considered $[3,8]$. Treatment of fistula's infections is required before surgery to avoid impaired drainage. Treatment of fistula's infections includes using a specific antibiotics or irrigation through the fistula with normal saline then iodine solution diluted with normal saline to eliminate the infection $[4,7,15]$.

\section{Treatment of the OAF}

Our chief goal in the closure of the OAC is to prevent oral bacteria and food debris from perforating the sinus and causing sinus areolation, congestion, sinusitis, and impaired ciliary function [14]. Two important factors should be considered during the closure of OAF. Firstly, the maxillary sinus should be free of infection with proper nasal drainage. Secondly, the closure should be a healthy vascularized, wide-base, tension-free, soft tissue flap above the intact bone [15]. The immediate closure of an acute oroantral defects has been associated with a high success degree approximately $95 \%$. However, a secondary closure has reported a success rate of $67 \%$ [1].

Management of OAC can be classified into pharmacological, surgical, and non-surgical interventions [2, 8]. The surgical options for the treatment of the OAF can be categorized as autogenous, allogenous, xenografts, and synthetic/metals [3]. (Fig.1) Allogenous grafts include GTR using allogenous barrier membrane and the lyophilized fibrin glue and membrane which has also been used in the treatment of OAC [8]. Xenografts associated with flap closure such as procaine collagen membrane, lyophilized porcine dermis, bovine bone, and GTR using bovine barrier membranes is also suggested for the treatment of OAC [8]. Other methods are also used for the treatment of OAC such as gingival suturing, re-implantation of the third molar, hydroxylapatite blocks, metal plates, and haemostatic gauze [8]. Autogenous surgical treatment can be classified as bone grafts and soft tissue flaps [3]. Grafts are usually required for the closure of chronic fistula when the soft tissue flaps fail. Grafts can be taken from zygoma, retromolar area, chin, inter-septal and inter-radicular area, iliac crest, plasma-rich fibrin membrane, cryoplatelet gel, and septal cartilage. These grafts have been reported for the closure of OAC [8].

Soft tissue flaps can be either distant or local flaps [3]. Some of the classic techniques in soft tissue flaps are the buccal advanced flaps, palatal rotational flaps, palatal transitional flaps, and tongue flaps [8]. Tongue flaps offer a nutritious blood supply and great flexibility for reconstruction in different areas including palatal or oroantral fistulas, lip, and cheek [16]. This flap can be formed from dorsal, ventral, or lateral area of the tongue [16]. However, the lateral tongue is suggested to be the most suitable flap for the closure of the OAC [6]. The selection of the tongue flap depends on the location of the defect [16]. Common disadvantage of this flap are the necessity for general anesthesia [16]. However, a local anesthesia can be performed during the cutting of the pedicle 2 weeks after attachment [16]. Flap procedure is considered as an alternative option if the suturing alone is not enough for the closure of the fistula. ${ }^{3}$ Local soft tissue flaps such as pedicled buccal fat pad flap (PBFPF), buccal flaps $(\mathrm{BF})$ and palatal flaps $(\mathrm{PF})$, are considered an excellent treatment in the closure of most $\mathrm{OAF}[2,3]$. 


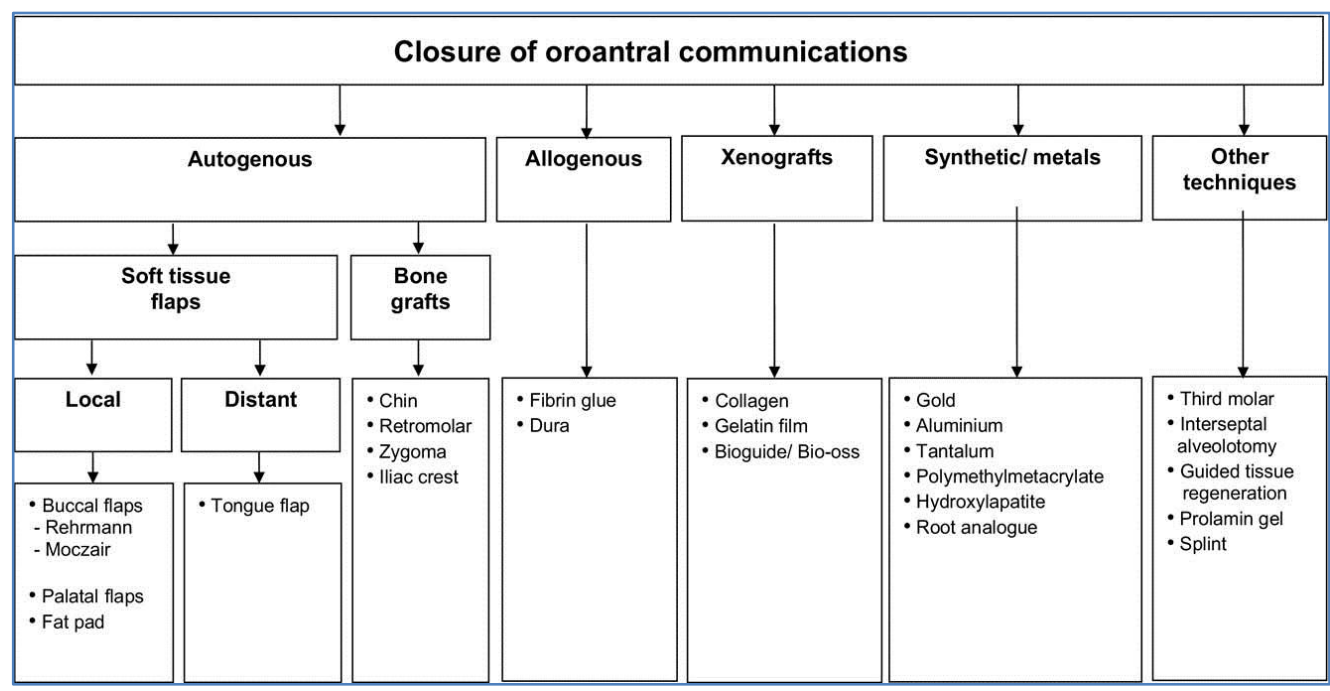

Fig-1: A summary of the treatment options for the oroantral communication [16]

\section{1- Buccal flaps}

The buccal flap was described by Axhausen in 1930 for the closure of small to moderate fistula [12]. This flap requires a buccally vertical incision with a thin layer of buccinator muscle to cover the fisula [4]. Although these flaps are simple to perform, it also requires careful manipulation as they are thin [5]. One of the advantages of this flap that it can be used in patients with severe alveolar resorption [4]. Different buccal mucoperiosteal flaps have been introduced including: advancement, rotated, transversal and sliding flaps [5].
Buccal advanced flap (BF) was introduced by Rehrman in 1936. It is the oldest and the most commonly used technique in the treatment of the OAF [3]. Many surgeons choose this technique as the first line of treatment for the closure of small communication or a minor fistula, which requires a simple suturing [5, 14]. This technique requires an excision of the epithelialized margins and development of 2 diverging vertical incisions extending to the buccal vestibule from the extraction site, then the broad-based trapezoid mucoperiosteal flap is elevated and placed over the defect followed by suturing the area from the buccal mucosa to the palatal mucosa using horizontal mattress sutures [3, 13-15] (Fig.2).

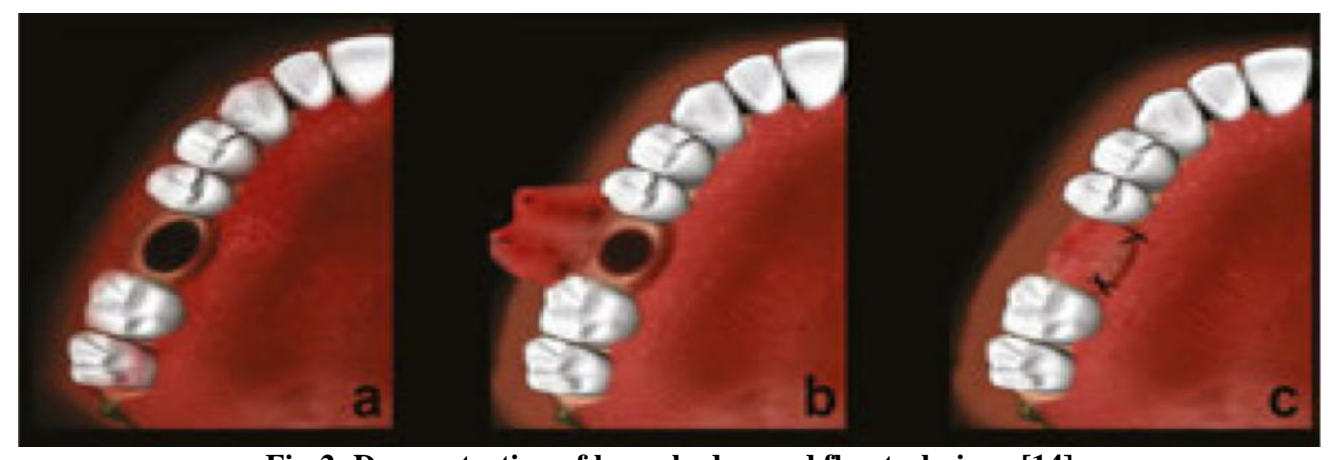

Fig-2: Demonstration of buccal advanced flap technique [14]

The advantages of this flap include a high survival rate and a sufficient blood supply [3]. Even though the buccal flaps are the most commonly used technique it also poses a major disadvantage, in which the buccal sulcus depth might be reduced after surgery, resulting in decrease of retention and discomfort in patients wearing dentures [3]. Weak perfusion of the flap is also considered a major disadvantage which may lead to improper closure of large defects [14].

The buccal sliding flap was introduced by Moczair, this flap is considered as an alternative technique for the closure of the alveolar fistula [3]. This technique has the advantage that the effect of buccal sulcus depth is minimal by shifting the flap one tooth distally [3, 5]. (Fig.3) However, the need for a significant amount of dentoalveolar detachment to facilitate the shift can lead to the onset of periodontal disease and gingival recession $[3,5]$.This procedure is considered more appropriate for the edentulous patient. However, Both of Rehrman and Moczair flap may result in swelling due to reflection of mucoperiosteal flap $[5,14]$. 

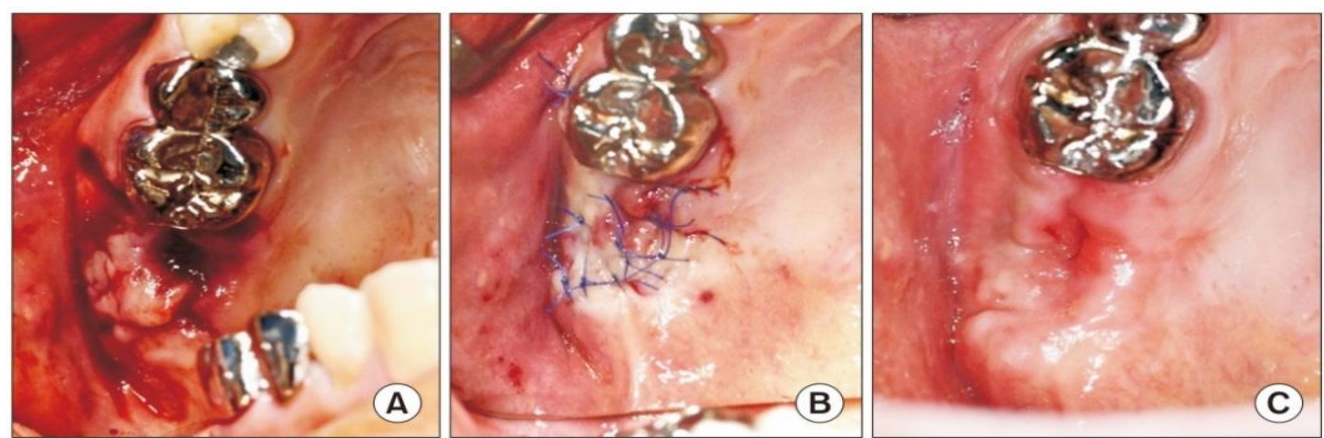

Fig-3: A: Represents a fistula in the second molar region of the maxilla. B: Covering the defect with buccal sliding flap and suturing with the palatal mucosa. C: After 3 weeks a complete healing can be seen [3]

\section{2- Buccal fat pad flap}

The buccal fat pad (BFP) is a fatty tissue surrounded by a thin fibrous capsule in a lobulated form which is located in the oromaxillofacial region inside both masticatory spaces $[3,18]$. It was first reported in 1732 by Heister as a glandular structure, and then reidentified as a fatty tissue in 1802 by Bichat [17]. This flap obtains its blood supply from the transverse division of the superficial temporal artery, buccal and deep temporal divisions of the maxillary artery, and branches of the facial artery [3]. Egyedi was the first to report the usage of BFP as pedicled grafts to close the
OAF, this flap has become more popular after the Tideman's study which reveals that the BFP is epithelialized within 3-4 weeks [3, 14].

This technique is similar to the buccal flap. First, a circular incision is made around the fistula. Next, a 2 vestibular incision is developed in the maxillary tuberosity area with a blunt dissection beneath the periosteum of the buccal flap and a light grip of the fat pad to the recipient site followed by suturing the fat pad to the palatal tissue with the fat left exposed in the oral cavity (Fig.4) [3, 14].

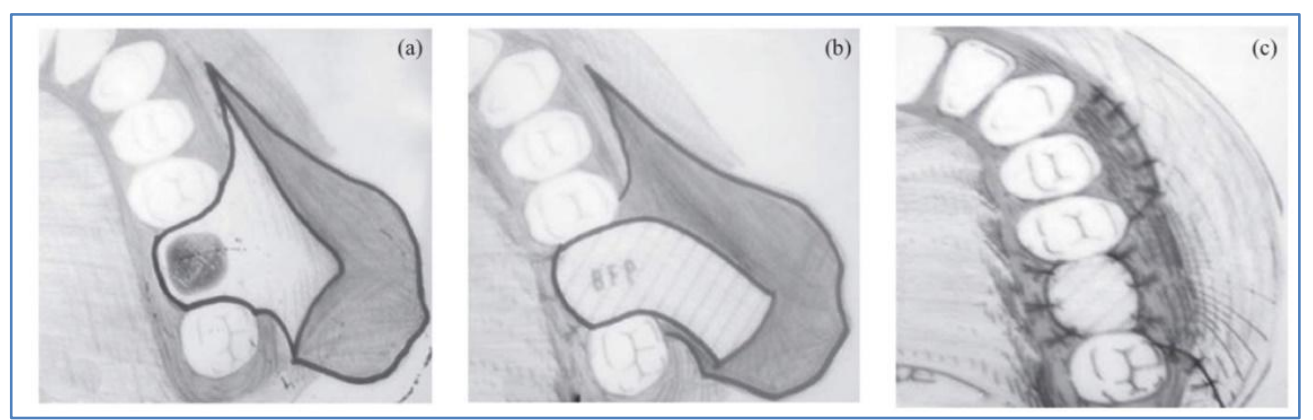

Fig-4: A- Incision and mobilization of the buccal flap. B- BFP moved and placed over the defect. C- BFP is sutured above the fistula

The advantages of this flap include sufficient blood supply, high success rate, decrease the risk of infection, rapid epithelialization of the uncovered fat, minimal donor site morbidity, and a great ability of utilization which make it more preferable for the closure of medium-sized defects of the maxilla $[3,6$, $11,14]$. However, when it is used for large defects a recurrent fistula and a graft necrosis may develop [3]. A lot of considerations should be obtained with this technique including the patient's history of radiation therapy, since the degree of previous radiation can affect the size and mobility of the fat pad [3]. Additionally, some surgeons consider this flap as an insecure technique due to the risk of traumatizing the pterygomaxillary space [11].

\section{3- Palatal flap}

The Palatal flap has a variety of forms that can be categorized as rotation-advancement, straightadvancement, hinged, Pedicle Island, anteriorly based, submucosal soft tissue pedicle, and submucosal island flaps [14].

Straight-advancement flap is usually required for the closure of minor palatal or alveolar defect because it does not offer much greater mobility for lateral coverage [5]. The use of the pedicle island flap has also been reported for the closure of OAF by Hendersen in 1974. A pedicle island flap is a one-stage procedure that supplies the flap with an excellent blood supply, bulk and mobility [5]. Many surgeons choose this flap to close the oroantral fistula due to its simplicity, versatility and mobility of the palatal island flap [5].

A modified submucosal connective tissue flap has also been reported for the closure of the OAF in the second and third molar area, this flap is known by its elasticity, ease of manipulation, and adaptation which contributes in preventing the folding and dog-ear 
formation [14]. In addition, this flap has the ability to prevent the problem of bone exposure at the donor site by dividing the flap into superior mucosal layer and underlying connective tissue layer [14].

This technique requires an excision of the wall of the fistula and the curette of the granulation tissue, then a development of an H-type window-like incision in the palatal mucosa about $4 \mathrm{~mm}$ from the gingival margins, followed by a dissection of the arterial connective tissue flap, and the positioning of the flap through the palatal tunnel maneuver and finally suturing the flap without any tension [14] (Fig.5).

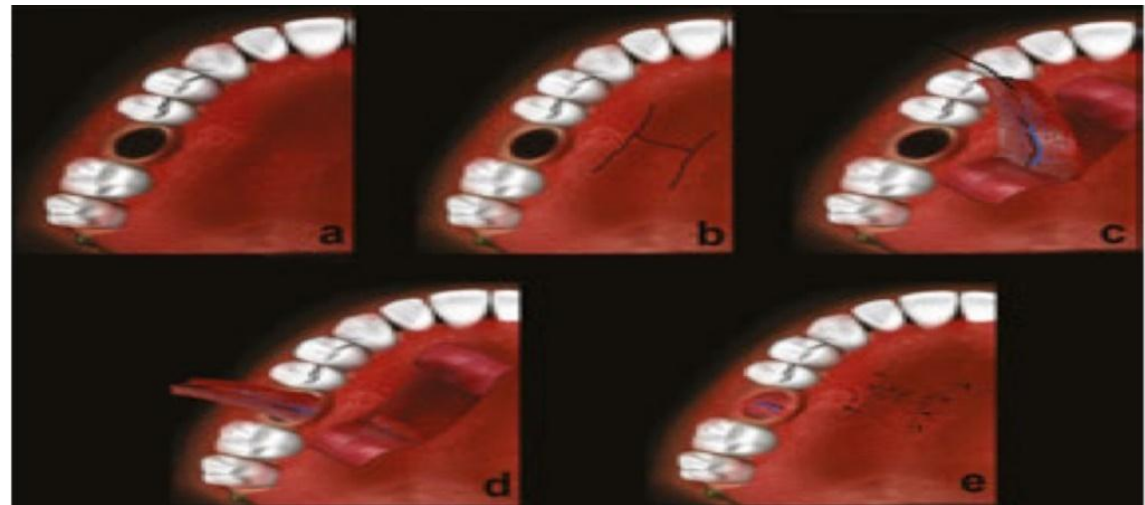

Fig-5: A- Excision of the fistulas wall. B- Development of $\mathrm{H}$ incision. $\mathrm{C}$ - $\mathrm{H}$ window-like flap and dissection of the arterial connective tissue flap. D- Placement of the flap through the palatal tunnel maneuver. E- Suturing the flap [14]

A common advantage of this flap over the full thickness flap is that the epithelial layer of the flap can be placed back to the donor site, which contributes to early healing of the wound and minimal discomfort for the patient [5]. Healing usually occurs at the donor site within 1 month [14].

The disadvantages of this flap are the possibility of injuring the blood supply, difficulty of the dissection, and the need for a skillful surgeon [14].

Full-thickness palatal rotational flap (PRF) is a widely used technique that requires a full-thickness mucoperiosteal flap in the treatment of large OAF that is more than $10 \mathrm{~mm}$ or undergoing a late repair [3]. This technique is considered more favorable to buccal flap in case the buccal vestibule depth needs to be preserved [3].

The flap design is arranged based on the greater palatine artery. It may require a circular excision of the fistula tract if needed, and development of a wide full-thickness flap carrying the greater palatine artery followed by rotating the flap to cover the defect [13, 14]. (Fig.6) Suturing with collagen sponge or palatal splint can be done to increase the secondary epithelialization of the donor site [3].
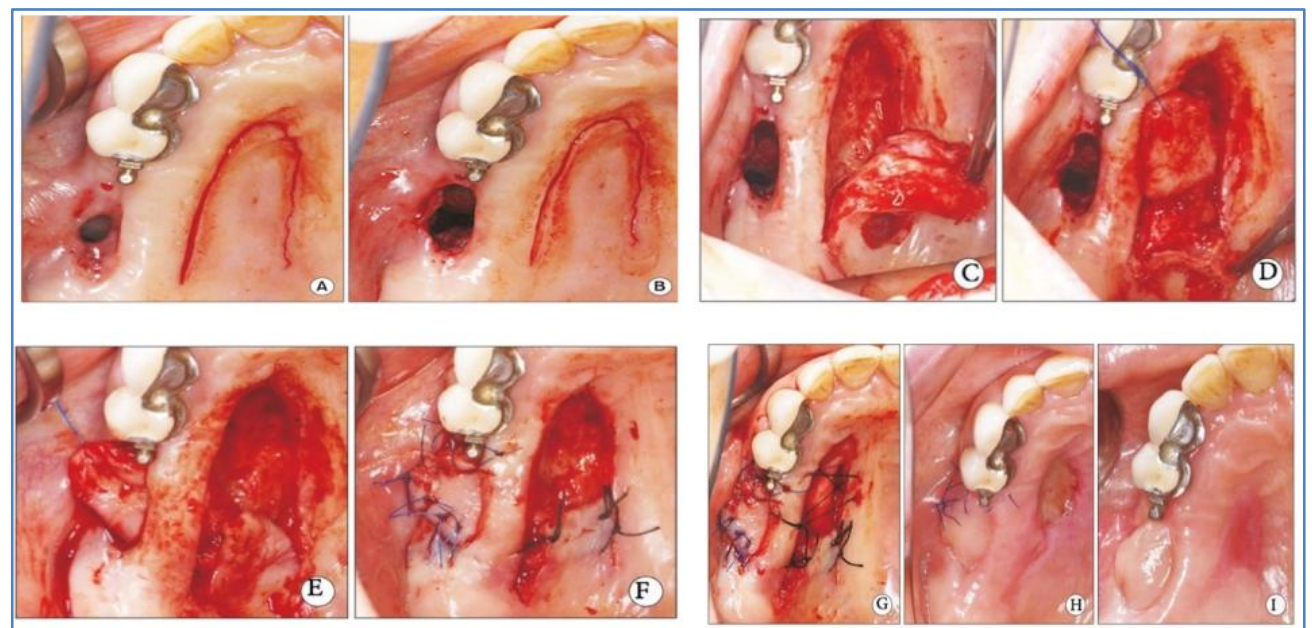

Fig-6: A, B-The circular excision of the fistulas wall reveals a larger opening than the suggested from the outside. C-After the incision, a full thickness palatal harvest carrying the greater palatine artery is raised. D- Dissection of the subepithelial region of the rotating flap. E- Rotation of the flap and placing it through the fistula's tract underneath the tunnel. F- Suturing the flap from the buccal and palatal area. G-Collagen sponge is used in the uncovered donor area. H- Secondary wound healing can be seen at the donor site. I- After 2 months, a complete healing can be observed [3]. 
The most important key points in determining the width of the flap are the angle of rotation and the bone defect [14]. The advantages of this flap are the remarkable thickness, keratinized tissue, sufficient vascularization, and the preservation of the vestibular depth [3, 14]. However, in case of thick keratinized tissue it may limit the rotation if the fistula is located in the maxillary tuberosity [14]. The common disadvantages are restriction in the movement of the flap to be rotated due to the greater palatine artery, and a possible kinking or dog-ear formation at the pivot point due to flap rotation which may affect the vascular supply. In case a dog ear formation is present, it must be excised to acquire a better adaptation [3, 14]. Sometimes, a re-epithelization should be considered due to the exposed bone structure of the hard palate [14]. In medically compromised patients, the risk of necrosis of the exposed bone in the donor site may be present. ${ }^{14}$ Kruger recommended a V-shaped excision in the region of the greatest bend in the flap to prevent wrinkling and folding [14]. Epithelialization (secondary wound healing) often take place at the exposed palatal bone of the donor site within 2 weeks [3]. A Palatal stent is suggested after palatal rotational flap to stabilize the flap and reduce the edema [14].

\section{Post-operative assessment}

Post-operative management is recommended to maintain adequate healing and proper closure of the communication. Analgesics such as, nonsteroidal antiinflammatory drugs $\left(\mathrm{NSAID}_{\mathrm{S}}\right)$, soft foods diet, and nasal decongestants are suggested postoperatively [12]. Antibiotics such as amoxicillin and clavulanic acid (Amoxyclav/Augmentin) should be prescribed as $1 \mathrm{~g}$ twice a day, or $300 \mathrm{mg}$ of clindamycin 3 times daily for a minimum 5 day [15]. The patient is instructed to drink from the opposite side to avoid trauma to the operated site. Nose blowing and sneezing while the mouth is closed should be avoided for 2 weeks. A warm saline mouth rinse should be used to clean the wound area. Smoking and using straw should be avoided. Physical activities that increase intra-sinusoidal pressure is avoided until the healing is completed [15]. After surgery, the patient should not move his tongue over the sutured line of the flap for 7 days [15].

\section{SUMMARY AND CONCLUSION}

After an extraction in the posterior region of the maxilla, the patient may experience unusual discomfort associated with postnasal exudate and halitosis which may indicate the presence of OAC [3]. Clinical examination and follow-up should be considered if the surgeon suspected the presence of OAC. Radiographs such as panoramic and waters view should be performed to validate the presence of sinus infection [3]. Cone-beam computed tomography (CBCT) is also used to confirm the presence of any connection between the maxillary sinus and the oral cavity [3]. Elimination of sinus infection should be the priority before any surgical intervention [3]. Infections can be managed by medication and sinus irrigation in case of acute sinusitis [3]. However, in the presence of chronic sinusitis with severe infection, ESS and Caldwell-Luc procedures can be performed before the closure of OAF [3]. A spontaneous healing is expected if the fistula is less than $2 \mathrm{~mm}$ with no sinus infection. However, in case the fistula is larger than $3 \mathrm{~mm}$, a surgical intervention should be considered for a better outcome.

Buccal flap, buccal fat pad flap, and Palatal flap have indicated a successful result in the closure of most OAF. Buccal flaps are the most commonly used technique for the treatment of OAF. A high survival rate and sufficient blood supply are the main advantages of this flap. However, the decrease in buccal sulcus depth after surgery is considered the major disadvantage of this technique.

BFPF is a fatty tissue surrounded by a thin fibrous capsule. This technique is often used for the closure of medium-sized defects. Common advantages of this flap are the nutritious blood supply, minimal donor site morbidity, and a great ability of utilization. However, a recurrent fistula and graft necrosis may develop in case of larger defects, thus it should be covered and sutured with the oral mucosa as a double layer [3].

Palatal flaps are a widely used technique in the closure of OAF. This technique is recommended for large OAF which is more than $10 \mathrm{~mm}$ or undergoing a late repair. Palatal rotational flap is suggested for the repair of larger defects. The advantages of this flap are the remarkable thickness and sufficient vascularization. Restriction in the movement of the flap due to the greater palatine artery is considered the major disadvantage of this technique.

The following reasons may lead to failure after the closure of the OAC/OAF [15]

1. Impaired blood supply caused by excessive tension on the flap which will prevent healing.

2. Inadequate pre-operative antibiotic therapy and irrigation for an existing sinus infection or disease.

3. Improper excision of epithelialized margins and improper trimming of bony margins before the closure.

4. Post-operative instruction was not given correctly or by some negligence from the patient side.

\section{REFERENCES}

1. Yalçın, S., Öncü, B., Emes, Y., Atalay, B., \& Aktaş, İ. (2011). Surgical treatment of oroantral fistulas: a clinical study of 23 cases. Journal of Oral and Maxillofacial Surgery, 69(2), 333-339. 
2. Mourão, C. F. D. A. B. (2019). Which treatments are best for oro-antral fistulae?. Evidence-based dentistry, 20(2), 44-45.

3. Kwon, M. S., Lee, B. S., Choi, B. J., Lee, J. W., Ohe, J. Y., Jung, J. H., ... \& Kwon, Y. D. (2020). Closure of oroantral fistula: a review of local flap techniques. Journal of the Korean Association of Oral and Maxillofacial Surgeons, 46(1), 58-65.

4. Gheisari, R., Zadeh, H. H., \& Tavanafar, S. (2019). Oro-Antral Fistula Repair with Different Surgical Methods: a Retrospective Analysis of 147 Cases. Journal of Dentistry, 20(2), 107.

5. Awang, M. N. (1988). Closure of oroantral fistula. International journal of oral and maxillofacial surgery, 17(2), 110-115.

6. Batra, H., Jindal, G., \& Kaur, S. (2010). Evaluation of different treatment modalities for closure of oroantral communications and formulation of a rational approach. Journal of maxillofacial and oral surgery, 9(1), 13-18.

7. El-Hakim, I. E., \& El-Fakharany, A. M. (1999). The use of the pedicled buccal fat pad (BFP) and palatal rotating flaps in closure of oroantral communication and palatal defects. The Journal of Laryngology \& Otology, 113(9), 834-838.

8. Krishanappa, S. K. K., Eachempati, P., Nagraj, S. K., Shetty, N. Y., Moe, S., Aggarwal, H., ... \& Group, C. O. H. (2018). Interventions for treating oro- antral communications and fistulae due to dental procedures. The Cochrane Database of Systematic Reviews, 2018(8).

9. Yih, W. Y., Merrill, R. G., \& Howerton, D. W. (1988). Secondary closure of oroantral and oronasal fistulas: a modification of existing techniques. Journal of oral and maxillofacial surgery, 46(5), 357-364.
10. Hernando, J., Gallego, L., Junquera, L., Villarreal, P. (2010). Oroantral communications. A retrospective analysis. Med Oral Patol Oral Cir Bucal, 15(3):e499- e503.

11. Borgonovo, A.E., Berardinelli, F.V., Favale, M., Maiorana, C. (2012). Surgical options in oroantral fistula treatment. Open Dent J, 6:94- 98.

12. Parvini, P., Obreja, K., Begic, A. (2019). Decisionmaking in closure of oroantral communication and fistula. Int J Implant Dent, 5(1):13.

13. Dym, H., Wolf, J.C. (2012). Oroantral communication. Oral Maxillofac Surg Clin North Am, 24(2):239- ix.

14. Guhan, D., Yusuf, E., Cagrı, D., \& Gokhan, G. (2016). 'Management of the Oroantral Fistula', in Mohammad Hosein Kalantar Motamedi (ed.) A Textbook of Advanced Oral and Maxillofacial Surgery Volume 3. Turkey: Mohammad Hosein Kalantar Motamedi, 365-385.

15. Khandelwal, P., \& Hajira, N. (2017). Management of oro-antral communication and fistula: various surgical options. World journal of plastic surgery, 6(1), 3.

16. Visscher, S.H., Van, M.B., Bos, R.R. (2010). Closure of oroantral communications: a review of the literature. J Oral Maxillofac Surg, 68(6):13841391.

17. Bouloux, G. F., Steed, M. B., \& Perciaccante, V. J. (2007). Complications of third molar surgery. Oral and Maxillofacial Surgery Clinics, 19(1), 117-128.

18. Manuel, S., Kumar, S., \& Nair, P. R. (2015). The versatility in the use of buccal fat pad in the closure of oro-antral fistulas. Journal of maxillofacial and oral surgery, 14(2), 374-377. 